\title{
Cistoadenocarcioma Mucinoso de Ovário e Esclerose Sistêmica $\left.{ }^{*}\right)$
}

\section{Ovarian Mucinous Cystadenocarcinoma and Systemic Sclerosis}

\author{
Maíta Poli de Araújo ${ }^{(1)}$, Alfredo Carlos Simões Dornelas de Barros ${ }^{(2)}$, Marcelo Alvarenga Calil ${ }^{(3)}$, \\ Cláudio Roberto Bianco de Carvalho ${ }^{(4)}$, Lourdes Alves Cepeda ${ }^{(5)}$, Fausto Motta Ferraz ${ }^{(4)}$, \\ Rodrigo Ambar Pinto ${ }^{(4)}$, Thais Villela Peterson ${ }^{(4)}$ e Virginia Fernandes Moça Trevisani( ${ }^{(6)}$
}

\section{RESUMO}

O câncer de ovário é a terceira causa de morte entre as neoplasias malignas em mulheres e o cistoadenocarcinoma mucinoso é uma neoplasia maligna originária do epitélio ovariano e que pode adquirir grandes dimensões. Alguns estudos sugerem um aumento do risco de neoplasia em pacientes com esclerose sistêmica (ES), mas a associação com neoplasia de ovário, principalmente do subtipo mucinoso, não é comum. Os autores descrevem um caso de cistoadenocarcinoma mucinoso de ovário em uma mulher branca de 52 anos com diagnóstico de ES difusa há seis anos, tratada previamente com D-penicilamina e ciclofosfamida, que procurou o serviço médico com queixa de dor e aumento do volume abdominal há três meses. Ao exame físico apresentava facies esclerodérmica, membros superiores com diminuição da elasticidade e enxerto bitibial em membros inferiores; no abdome havia a presença de uma massa endurecida estendendo-se da região suprapúbica até o epigástrio. Foi realizada laparotomia exploradora onde se encontrou uma massa cística no ovário direito, com peso de 3.300 gramas. Em seguida procedeu-se exérese do tumor com histerectomia total e ooforectomia bilateral. O exame histológico confirmou tratar-se de um cistoadenocarcinoma mucinoso sem invasão de cápsula.

Palavras-chave: esclerose sistêmica, câncer de ovário, ciclofosfamida.

\begin{abstract}
Ovarian cancer is the third cause of death among malignant neoplasia in women, and the mucinous cystadenocarcinoma is a malignant neoplasia of the ovarian epithelium, which may achieve great dimensions. Some studies suggest an increased risk of neoplasia in patients with systemic sclerosis (SSc), but the association with ovarian neoplasia, specially the mucinous subtype, is rare. The authors describe a case of ovarian mucinous cystoadenocarcinoma in a 52 year-old Caucasian woman who had the diagnosis of SSC 6 years ago, previously treated with cyclophosphamide and D-penicillamine, who sought for a medical service complaining of pain and increase of abdominal volume during the last three months. At the physical examination, she presented sclerodermic facies, upper limbs with decreased elasticity and bitibial graft in the lower limbs; in the abdomen, a hardened mass extending to the suprapubic region up to the epigastric was observed. Exploratory laparotomy was performed and a cystic mass was found in the right ovary, weighing 3,300 grams. A surgical removal of the tumor with total hysterectomy and bilateral oophorectomy was then performed. Histological examination confirmed the diagnosis of mucinous cystadenocarcinoma without capsule invasion.
\end{abstract}

Keywords: systemic sclerosis, ovary cancer, cyclophosphamide.

\footnotetext{
Trabalho realizado nas Disciplinas de Ginecologia e Reumatologia da Faculdade de Medicina da Universidade de Santo Amaro, São Paulo, Brasil. Recebido em 06/11/03. Aprovado, após revisão, em 19/01/04.

1. Médica residente da Disciplina de Ginecologia e Obstetrícia. Faculdade de Medicina da Universidade de Santo Amaro.

2. Professor Titular da Disciplina de Ginecologia. Faculdade de Medicina da Universidade de Santo Amaro. Presidente da Sociedade Brasileira de Mastologia.

3. Professor Titular da Disciplina de Obstetrícia. Faculdade de Medicina da Universidade de Santo Amaro.

4. Acadêmico. Faculdade de Medicina da Universidade de Santo Amaro.

5. Médica Anatomo-Patologista. Faculdade de Medicina da Universidade de Santo Amaro.

6. Professora Titular da Disciplina de Reumatologia e Imunologia. Faculdade de Medicina da Universidade de Santo Amaro.

Endereço para correspondência: Maíta Poli de Araújo. Al. Joaquim Eugenio de Lima, 1601, ap. 84, CEP 01403 003, São Paulo, SP. E-mail: aja.ops@zaz.com.br
} 


\section{INTRODUÇÃO}

O câncer de ovário é a terceira causa de morte entre as neoplasias malignas em mulheres e em razão de sua discreta sintomatologia, as pacientes procuram auxílio médico geralmente em estágios avançados, piorando o prognóstico da doença ${ }^{(1)}$. O cistoadenocarcinoma mucinoso é uma neoplasia maligna originária do tecido epitelial do ovário e que freqüentemente adquire grandes dimensões ${ }^{(1)}$.

Alguns estudos sugerem aumento do risco de neoplasias em pacientes com esclerose sistêmica (ES), principalmente de pulmão e mama ${ }^{(2-4)}$. Este risco estaria relacionado com fatores clínicos e epidemiológicos (idade, sexo), imunológicos (presença de anticorpos anticentrômero e anti Scl-70) e histológicos (fibrose pulmonar) ${ }^{(2,5)}$. A associação entre ES e neoplasia de ovário, principalmente do subtipo mucinoso, é incomum ${ }^{(6)}$, motivo pelo qual justifica-se o relato deste caso.

\section{RELATO DE CASO}

MLEL, 52 anos, branca, viúva, procurou serviço médico com queixa de dor e aumento do volume abdominal há três meses. A paciente referia diagnóstico de esclerose sistêmica (ES) difusa há seis anos, através das manifestações clínicas, da biópsia cutânea e da capilaroscopia periungueal com padrão SD. Durante os três primeiros anos de seguimento realizou tratamento com D-penicilamina e ciclofosfamida. No começo a doença foi agressiva, necessitando de várias internações em conseqüência do comprometimento sistêmico, com envolvimento do trato gastrintestinal, pulmonar e vascular, chegando a realizar enxerto de pele em membros inferiores em razão de úlceras cutâneas.

A paciente inicialmente foi tratada com D-penicilamina. Com seis meses de seguimento, apresentou alteração na prova de função pulmonar (padrão restritivo moderado) e padrão de vidro despolido em bases pulmonares na tomografia computadorizada, sugerindo alveolite. Foi iniciado tratamento com pulsoterapia mensal de ciclofosfamida por seis meses. Com a estabilização do quadro pulmonar, retornou o tratamento com D-penicilamina.

Nesse mesmo período, em acompanhamento na ginecologia, realizou ultra-som pélvico que evidenciou imagem anecóica, regular, bem delimitada em ovário direito. A paciente não fez acompanhamento clínico ou ultra-sonográfico após este achado até o aparecimento dos sintomas abdominais e há três anos não fazia uso de qualquer medicação para a ES. Relatava menarca aos 13 anos, com ciclos eumenorréicos, uso de anticoncepcional oral por nove anos e menopausa há três anos. Com relação aos antecedentes obstétricos era quintigesta, quintípara, com partos normais sem intercorrências.

Ao exame físico apresentava facies esclerodérmica com nariz afilado, microstomia e pregueamento perilabial (figura 1); abdome globoso, flácido, indolor à palpação, com a presença de uma massa de consistência endurecida, multinodular, estendendo-se da região suprapúbica até o epigástrio; os membros superiores evidenciavam dedos cilíndricos com atenuação dos relevos e pregas interdigitais e diminuição da elasticidade da pele; nos membros infe-

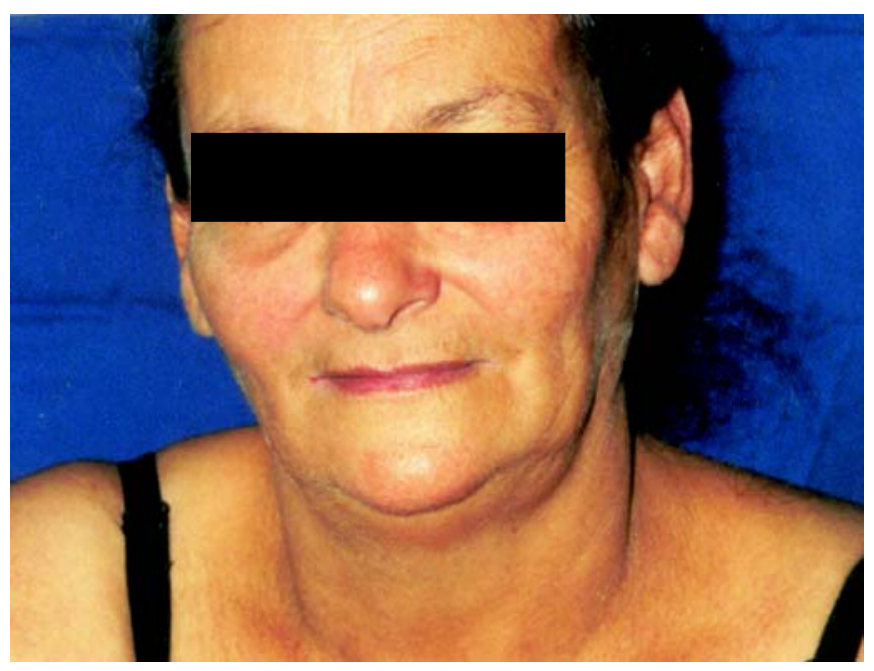

Figura 1 - Facies esclerodérmica.

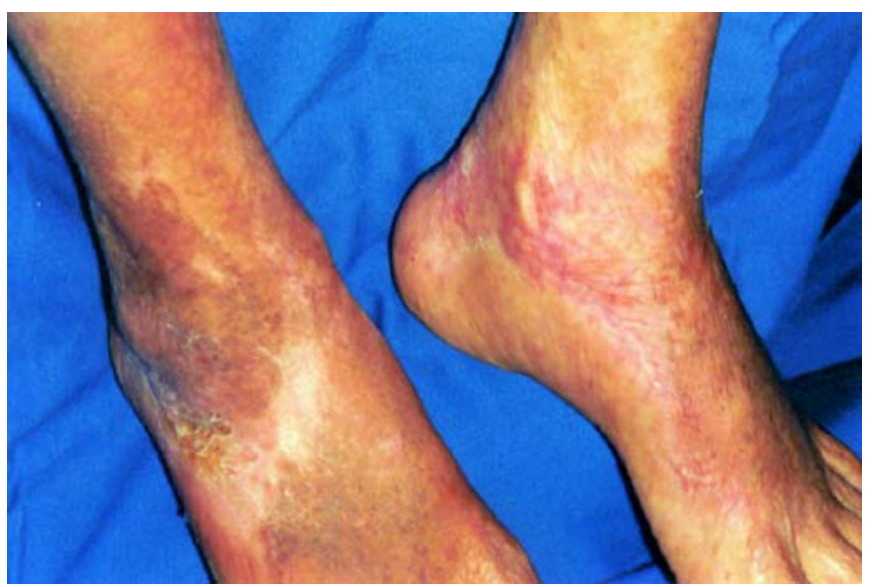

Figura 2 - Membros inferiores com enxerto. 
riores, observava-se enxerto de pele na região pré-tibial, com lesões cutâneas hiper e hipocrômicas (figura 2).

Ao toque vaginal, observava-se útero de tamanho normal e massa anexial direita palpável com cerca de vinte centímetros de diâmetro. A ultra-sonografia pélvica evidenciou ovário direito com dimensões aumentadas, com a presença de múltiplos cistos de contornos regulares, hipoecogênicos, que mediam aproximadamente $\mathrm{N} 1=13,9 \mathrm{~cm}, \mathrm{~N} 2=7,0 \mathrm{~cm}$, $\mathrm{N} 3=6,8 \mathrm{~cm}, \mathrm{~N} 4=15,7 \mathrm{~cm}$ e $\mathrm{N} 5=10,5 \mathrm{~cm}$, nos seus maiores eixos (figura 3).

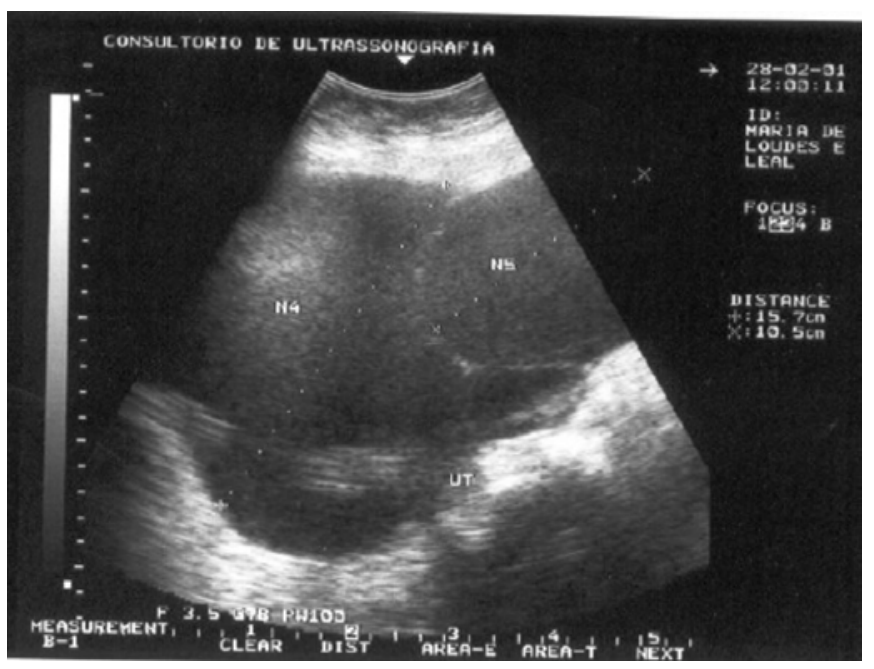

Figura 3 - Ultra-sonografia: presença de múltiplos cistos hipoecóicos em ovário direito.

Foi realizada laparotomia exploradora, constatando-se uma massa de contornos irregulares, consistência cística, no ovário direito, medindo $24 \times 15 \times 13 \mathrm{~cm}$, com peso de 3.300 gramas (figura 4). Em seguida procedeu-se à exérese do tumor, histerectomia total e ooforectomia bilateral. O lavado peritoneal foi negativo para células neoplásicas e a avaliação dos órgãos abdominais foi realizada minuciosamente, não sendo encontrado nenhum implante neoplásico.

O estudo anatomopatológico concluiu tratar-se de um cistoadenocarcinoma mucinoso de ovário direito, sem comprometimento capsular, e útero normal (figura 5).

A paciente evoluiu com melhora clínica e teve alta no segundo pós-operatório. Será acompanhada ambulatorialmente com dosagem periódica dos marcadores tumorais (CEA e Ca 125) e exame ginecológico de rotina.

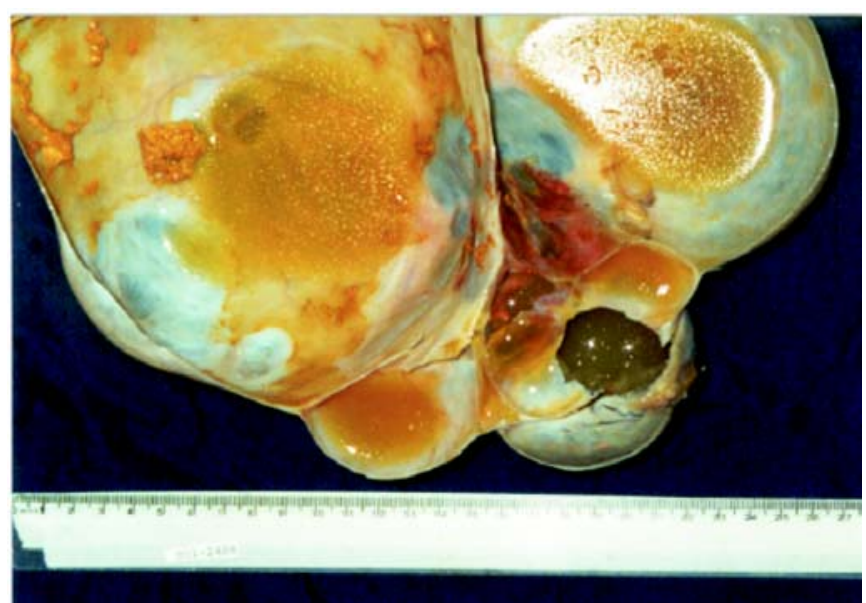

Figura 4 - Tumor de ovário direito, cístico, multiloculado.

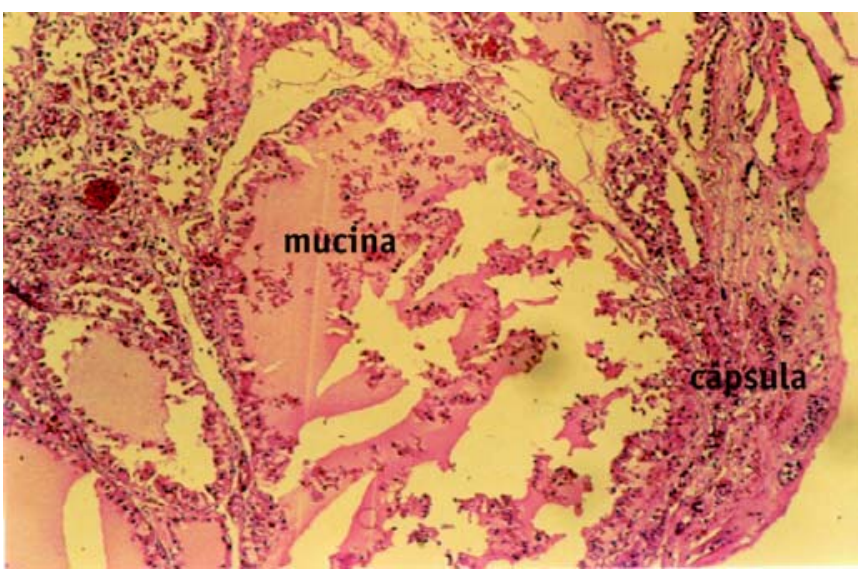

Figura 5 - 100X HE: Cápsula do ovário invadida pela neoplasia, mas sem ultrapassá-la.

\section{DISCUSSÃO}

O câncer primário do ovário é mais incidente em mulheres de nível sócio-econômico médio para alto, acima dos 50 anos, brancas e corresponde a $15 \%$ das neoplasias malignas nos serviços de ginecologia geral ${ }^{(1)}$. A etiologia destes tumores inclui vários fatores que suportam a hipótese de "ovulação incessante", na qual este tipo de neoplasia desenvolve-se em razão de uma aberração no processo de reparação cicatricial na superfície do epitélio ovariano, que sofre processo de rotura e reparação a cada ciclo ovulatório $^{(1)}$. Assim, haveria maior incidência em pacientes com menarca precoce e menopausa tardia, decorrente de atividade ovariana prolongada. Por outro lado, o uso de anticoncepcionais orais seria fator protetor e seu efeito 
permaneceria por vários anos, mesmo após a interrupção do uso. Neste relato, o período da menacma da paciente estava dentro da normalidade e ela fez uso de anticoncepcional oral por nove anos; mesmo assim, apresentou neoplasia maligna de ovário.

O tratamento dos tumores de ovário depende do estadiamento e é eminentemente cirúrgico seguido ou não da quimioterapia. No estádio I (tumor limitado a um dos ovários) procede-se a histerectomia total abdominal com anexectomia bilateral e omentectomia. Alguns autores indicam a omentectomia apenas nos estádios III e IV, pois acreditam que nos estádios I e II o procedimento não levaria à melhora da sobrevida ${ }^{(1)}$. A quimioterapia é indicada nos estágios avançados ou quando a cirurgia radical não é possível ou em caso de recidiva, de metástases ${ }^{(1)}$.

Alguns trabalhos indicam freqüência de 3\% a $7 \%$ de neoplasia nos pacientes com $\mathrm{ES}^{(2,4,5,7)}$. Historicamente, o câncer de pulmão é o que está mais associado à ES e isto estaria relacionado com a fibrose pulmonar que ocorre nestes pacientes e que poderia ser um fator predisponente ao desenvolvimento da neoplasia ${ }^{(2,3,8-10)}$.

Poucos são os relatos associando neoplasia de ovário e esclerose sistêmica ${ }^{(6)}$. A revisão da literatura entre 1965 e 2001 indica a presença de 12 casos de câncer de ovário, e embora na maioria dos casos predomine o tipo histológico adenocarcinoma, nenhum trabalho cita a presença do cistoadenocarcinoma mucinoso (Tabela 1). Os trabalhos indicam que a neoplasia pode aparecer poucos anos após o diagnóstico da ES ou depois de mais de dez anos ${ }^{(2)}$. Neste relato, o diagnóstico de certeza do cistoadenocarcinoma mucinoso de ovário foi realizado seis anos após a ES. A imagem ultra-sonográfica que a paciente apresentava no início da pulsoterapia não parecia sugestiva de neoplasia, tendo em vista o padrão anecóico e bem delimitado. $\mathrm{O}$ achado poderia corresponder a um cisto simples de ovário e o correto seria o acompanhamento semestral ultra-sonográfico e a solicitação de marcadores tumorais para excluir malignidade.

Com relação à etipatogenia das neoplasias em pacientes com ES, alguns autores acreditam que a positividade para o anticorpo anticentrômero poderia ser considerada um fator de risco, mas estudos maiores são necessários para confirmar este achado e avaliar outros riscos nestes pacientes ${ }^{(5)}$.

A utilização de medicamentos tais como a ciclofosfamida também poderia induzir ao câncer ${ }^{(3)}$. Atualmente sabe-se que a ciclofosfamida tem ação tóxica em diversos órgãos e que alguns pacientes que utilizam este medicamento por muitos anos podem desenvolver câncer de bexiga ${ }^{(11)}$. A etipatogenia deste poder carcinogênico é desconhecida, mas existem alguns trabalhos mostrando que este imunossupressor seria capaz de modificar o gene supressor de tumor p53, favorecendo o desenvolvimento de neoplasias ${ }^{(11)}$. A ciclofosfamida também apresenta toxicidade gonadal ${ }^{(11)}$. Estudos demonstram que a falência ovariana precoce é a

TABELA 1

FreqüÊNCIA DE CÂNCER DE OVÁRIO EM PACIENTES COM ES NO PERÍODO ENTRE 1965 E 2001

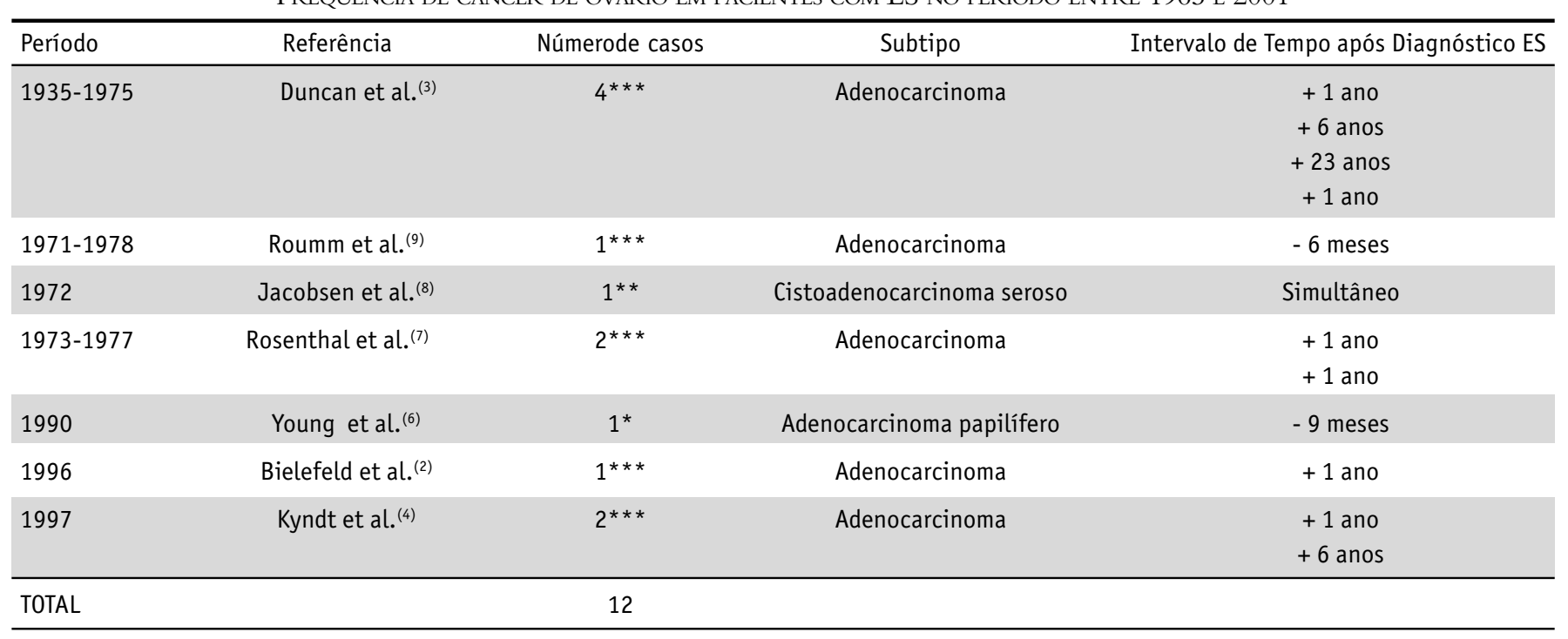

${ }^{*}$ : esclerose sistêmica limitada; ${ }^{* *}$ : esclerose sistêmica difusa; ${ }^{* * *}$ : esclerose sistêmica não determinada. 
principal consequiência da poliquimioterapia e esta citotoxicidade é irreversível e progressiva no ovário porque o número de células germinativas é limitado e fixo desde a vida fetal ${ }^{(11)}$. Secundariamente à toxicidade ovariana, a destruição folicular pode alterar a concentração de estradiol plasmático levando à diminuição do peso do útero ${ }^{(11)}$. Com o intuito de preservar a função ovariana em mulheres jovens, alguns autores defendem o uso de análogos de GnRH concomitante aos agentes alquilantes ${ }^{(11)}$.

Neste relato a paciente apresentava imagens sugestivas de comprometimento ovariano no ultra-som desde 1995, ano em que foi descoberta a ES e iniciada a utilização da pulsoterapia com ciclofosfamida. Assim, fica dificil saber se o uso deste medicamento poderia ter contribuído para o

\section{REFERÊNCIAS}

1. Abrão FS, Abrão MS: Câncer do ovário. 3a ed., São Paulo, Ed. Roca, 1997, p. 87-97.

2. Bielefeld P, Meyer P, Caillot D, et al: Sclerodermie systemique et cancers: 21 observations et revue de la litterature. Rev Med Interne 17: 810-3, 1996.

3. Duncan SC, Medsger TA: Cancer and systemic scleroderma. Arch Dermatol 115: 950-5, 1979.

4. Kyndt X, Hebbar M, Queyrel V, et al: Systemic scleroderma and cancer: Search for predictive factors of cancer in 123 patients with scleroderma. Rev Med Interne 18: 528-32, 1997.

5. Higuchi M, Horiuchi T, Ishibashi N, et al: Anticentromere antibody as a risk factor for cancer in patients with systemic sclerosis. Clin Rheumatol 19: 123-6, 2000. desenvolvimento de câncer em um ovário previamente alterado ou se a ES por si só seria capaz de modificar a estrutura ovariana do mesmo modo que ocorre em outros órgãos, produzindo fibrose maciça de elementos conjuntivos e endarterite proliferativa, e estas alterações seriam fatores predisponentes para o surgimento da neoplasia de ovário.

Em resumo, diversas neoplasias (principalmente de pulmão e mama) podem ocorrer nos pacientes com ES e a associação com o tumor de ovário é incomum. Tendo em vista que a neoplasia de ovário é uma das neoplasias silenciosas do organismo e de difícil diagnóstico, pacientes do sexo feminino, portadores de ES, devem ter acompanhamento rigoroso com ginecologista, para rastreamento de câncer de mama, útero e ovário.

6. Young R, Towbin B, Isern R: Scleroderma and ovarian carcinoma. Br J Rheumatol 29: 314-5, 1990.

7. Rosenthal AK, McLaughlin JK, Gridley G, Nyrén O: Incidence of cancer among patients with systemic sclerosis. Cancer 1: 910-4, 1995.

8. Jacobsen BB, Pedersen M, Herting SE: Generalized scleroderma. Report on a case associated with acute nephropathy, haemolytic anaemia and malignant ovarian tumour. Acta Med Scand 192: 10711, 1972.

9. Roumm AD, Medsger TA: Cancer and systemic sclerosis. An epidemiologic study. Arthritis Rheum 28: 1336-40, 1985.

10. Marques Neto JF, Provenza JR, Samara AM: Esclerose sistêmica progressiva e neoplasias. Rev. Bras. Reumat 21: 97-100, 1981.

11. Plowchalk DR, Meadows MJ, Mattison DR: Reproductive toxicity of cyclophosphamide in the C57BL/6N mouse: Effects on uterine structure and function. Reprod Toxicol 6: 423-9, 1992. 